\title{
Effect of surfactant on the evolution of bubble motion
}

\author{
Aleksandra Antonnikova ${ }^{1, *}$, Anna Usanina $^{1}$ \\ ${ }^{1}$ National Research Tomsk State University, 634050 Tomsk, Russia
}

\begin{abstract}
Results of an experimental study of the process of ascent of a single bubble of air in the presence of a surfactant are presented. An empirical dependence for drag coefficient of the bubble that emerges in a liquid containing a surfactant in the region of small Reynolds numbers has been obtained. Theoretical studies of the effect of surfactants on the velocity of motion of a bubble in a nonstationary regime have been carried out. A theoretical analysis of the effect of nonstationary forces on the dynamics of bubble rising in the nonstationary regime has been conducted.
\end{abstract}

\section{Introduction}

In two-phase flows containing deformable particles of a dispersed phase (liquid-drop, bubble flows) the properties of the interface, in particular the coefficient of surface tension, play an important role. One of the physical factors that affect the boundary conditions is the presence of a surfactant that can change the criteria for the motion of a particle of the dispersed phase significantly. On the free boundary of a bubble or drop moving in a liquid with a surfactant, tangential capillary Marangoni forces arise contributing to an increase in the particle drag coefficient. Problems arising in the study of the laws of motion and the deformation of bubbles (or drops) in a liquid containing a surfactant are formulated in [1-8]. These problems are associated with a variety of types of interfacial interactions and processes (deformation, break down, collision of bubbles, capillary effects, coagulation, heat exchange between phases, adsorption, adhesion). The understanding and adequate description of the dynamics of the behavior of two-phase systems taking into account the physical and chemical properties of the dispersed and dispersive phases is extremely difficult and requires new experimental data (empirical dependencies and criterial relationships) on elementary processes occurring at the phase boundary during their interaction. In particular, at the present time there is a limited amount of experimental data that allows estimating the effect of nonstationary effects on the parameters of particle motion in a flow in the presence of surfactants in a dispersion medium. Nonstationary effects (the force associated with the attached mass, the "hereditary" Basset force) transforms the differential equation of the particle motion into an integro-differential one that significantly complicates the solution of the problem of the nonstationary motion of a particle. An experimental study of the particle motion in a nonstationary regime is also difficult because of the small characteristic times and distances of the process.

\footnotetext{
*Corresponding author: leva@niipmm.tsu.ru
} 
The purpose of this paper is to investigate the laws of the ascent of a single air bubble in a liquid containing a surfactant.

\section{Experimental setup and study technique}

To investigate the process of the ascent of a single air bubble in a liquid containing a surfactant, an experimental method and setup were used that met the following requirements: providing the precise measurement of all the main parameters (linear dimensions of the process and the physical properties of the fluid: density, coefficient of dynamic viscosity); control over the change in the characteristics of the ascent of the bubble due to the use of multi-angle video.

The experimental setup consists of a vertically mounted transparent tank with planeparallel walls filled with a liquid solution, a device for generating bubbles, and a system for visualizing the ascent of a single bubble. Glycerin, water-glycerol solution and distilled water were used as a liquid; liquid soap was used as a surfactant. Mass fraction of surfactant in the liquid varied in the range $\mathrm{z}=(0.01-0.13) \%$.

Air bubbles were obtained by means of a syringe. A hollow aluminum tube with a diameter of $3 \mathrm{~mm}$ was fixed to the outlet hole of the syringe. At the end of the tube a replaceable socket with a diameter (0.4-7.0) $\mathrm{mm}$ located in lower part of the tank is assembled. The separation of the bubble from the outlet hole did not occur due to the change in pressure (on exposure to the air flow) but in a natural way, when the resultant of all forces exceeded zero.

The visualization system included the light sources (two $18 \mathrm{~W}$ fluorescent lamps) installed on the rear panel along the entire height of the tank, a digital video camera "Panasonic HDC-SD60" and a high-speed video camera "Citius C100". The first camera was used to determine the size of the bubble; the shooting field was $5 \times 5 \mathrm{~cm}$ with a twofold increase. The second one fixed the ascent velocity of the bubble. The video was taken with a spatial resolution of $384 \times 790$ pixels with camera speed of at least 300 frames per second and exposure time $(0.5-2.0) \mathrm{ms}$. To control the distance traveled by the bubble a scale rule was used with a division value of $1 \mathrm{~mm}$ that was calibrated taking into account the optical distortion due to the walls of the tank.

\section{Experimental results}

Experimental studies were carried out in the following range of parameters: bubble diameter $D=(0.82 \div 24) \mathrm{mm}$; the ascent velocity of the bubble $u=\left(3.9 \cdot 10^{-3} \div 0.22\right) \mathrm{m} / \mathrm{s}$; liquid density $\rho_{l}=(1000 \div 1260) \mathrm{kg} / \mathrm{m}^{3}$; coefficient of dynamic viscosity of liquid $\mu_{l}=\left(10^{-3} \div 2.44\right) \mathrm{Pa} \cdot \mathrm{s}$; Reynolds number $\mathrm{Re}=\left(3.5 \cdot 10^{-3} \div 211\right)$; the content of the surfactant $z=(0.01 \div 0.13) \%$.

The experimental data on the stationary ascent velocity of a single spherical bubble in the presence and without surfactant are compared. It is found that the velocity of a bubble emerging in a liquid with a surfactant is less than the velocity of a bubble rising in a clean liquid.

In the range of Reynolds number $\operatorname{Re}=3.5 \cdot 10^{-3} \div 0.6$ an empirical dependence for the drag coefficient of a spherical bubble rising in the presence of a surfactant is obtained: $C_{D}=24.06 / \mathrm{Re}$. The coefficient of determination of the obtained dependence is equal to $\mathrm{R}^{2}=0.99$. Calculations of the drag coefficient for nonspherical bubbles rising in the presence of a surfactant were also made. The equivalent spherical diameter was used as the diameter (Fig. 1, 2). 


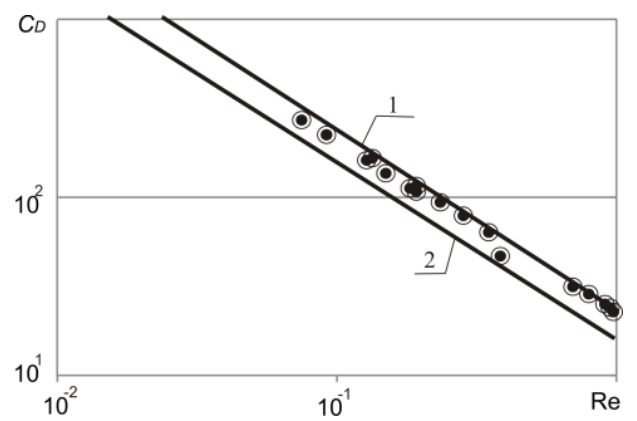

Fig. 1. Dependence of the drag coefficient of a nonspherical bubble on Reynolds number: $\bullet$ - with surfactants; 1 - Stokes curve (for a solid sphere); 2 - the Hadamard-Rybchinsky curve (for the liquid sphere).

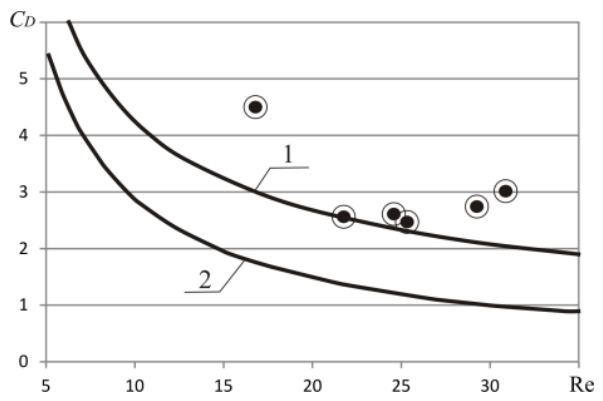

Fig. 2. Dependence of the drag coefficient of a nonspherical bubble on Reynolds number: $\bullet$ - with surfactants; 1 - Klyachko curve (for a solid sphere); 2 - curve $C_{D}=48 / \operatorname{Re}$ (for the liquid sphere).

Theoretical calculations of the bubble velocity taking into account the nonstationary forces (the forces associated with the attached mass and the "hereditary" Basset force) for a non-stationary ascent regime. A comparative analysis of the theoretical and experimental dependences has shown that at calculating the dynamics of the ascent of a single spherical bubble it is necessary to take into account nonstationary forces.

The contribution of the viscous drag force $F_{S t}$, the force associated with the attached mass $F_{a d}$, and the "hereditary" Basset force $F_{B}$ to the total drag force $F_{\Sigma}$ of a single spherical bubble rising in the presence of surfactant is estimated. The Basset force is calculated from the analytical formula of L.D. Landau [8]. Calculations of the forces acting on the bubble were carried out for the following experimental conditions: $D=2.33 \mathrm{~mm}$, $\rho_{l}=1218 \mathrm{~kg} / \mathrm{m}^{3}, \mu_{l}=0.099 \mathrm{~Pa} \cdot \mathrm{s}, \mathrm{Re}=1.15$. The forces acting on the bubble were calculated by the formulas:

$$
\begin{gathered}
F_{A}=\frac{\pi D^{3}}{6} \rho_{l} g, F_{a d}=\frac{\pi}{12} \rho_{l} D^{3} a, F_{s t}=-C_{D} \frac{\pi D^{2}}{4} \frac{\rho_{l}(a t)^{2}}{2}, \\
F_{B}=3 a D^{2} \sqrt{\pi \mu_{l} \rho_{l} t}, F_{\Sigma}=F_{B}+F_{s t}+F_{a d},
\end{gathered}
$$

where $F_{A}$ is the Archimedes force; $C_{D}=24.06 / \mathrm{Re}, a=0.18 \mathrm{~m} / \mathrm{s}^{2}$ is the bubble acceleration, calculated from the experimental data.

Calculations showed that the main contribution to the total drag force on the nonstationary region of the bubble trajectory is made by the viscous drag force and the "hereditary" Basset force (Fig. 3). At $t<t_{1} \approx 0.1 t *$ the value of the Basset force is 
insignificantly different from the drag force $\left(F_{B} \approx F_{S t}\right)$, and at $t_{1}<t<t *$ Basset force becomes less than the viscous drag force and at $t=t_{*}$ Basset force is equal to $F_{B} \approx 0.3 F_{s t}$. The force associated with the attached mass is $\sim 0.5 \%$ from the sum of all the forces acting on the bubble. It is constant on the non-stationary part of the bubble motion. A similar estimate of the forces for a bubble rising in a pure liquid showed a qualitative agreement of the results for a bubble rising in the presence and without surfactant.

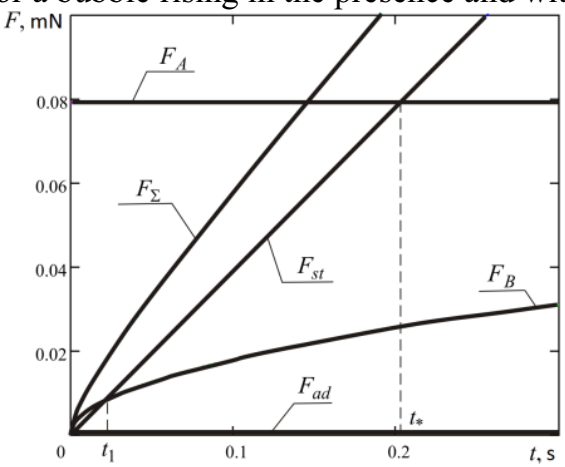

Fig. 3. Estimation of the forces acting on the bubble at uniformly accelerated motion.

\section{Conclusion}

The results of the experimental-theoretical study of the dynamics of the ascent of a single bubble of air in the presence of surfactant allow to make the following conclusions.

- The presence of surfactant leads to an increase in the drag coefficient of $33 \%$ at Reynolds number $\operatorname{Re}<1$ and approximately of $50 \div 60 \%$ at $\operatorname{Re}>1$ and, accordingly, to a decrease in the ascent velocity of the bubble.

- An empirical dependence for the drag coefficient of a single spherical bubble rising in a liquid in the presence of a surfactant for Reynolds numbers $\operatorname{Re}<1$ is obtained.

- The theoretical analysis of the problem of the bubble rising in liquid containing surfactants showed that the "hereditary" Basset force and the viscous drag force make the main contribution to the total drag force on the non-stationary region of the bubble rise.

The study was supported by the Russian Science Foundation (No 15-19-10014).

\section{References}

1. V.G. Levich, Physico-chemical hydrodynamics (Prentice-Hall, New Jersey, 1959)

2. A.N. Frumkin, V.G. Levin, Russian Journal of Physical Chemistry A, 21, 1183 (1947)

3. R. Clift, J.R. Grace, M.E. Weber, Bubbles, Drops and particles (Academic Press, London, 1978)

4. R. Griffith, Chem. Eng. Sci. 17, 1057, (1962)

5. R. Davis, A. Acrivos, Chem. Eng. Sci. 21, 681 (1966)

6. J. Harper, Appl. Sci. Res. 38, 343 (1982)

7. Y. Wang, D. Papageorgiou, C. Maldarelli, J. Fluid Mech. 390, 251 (1999)

8. L.D. Landau, E.M. Lifshits, Course of Theoretical physics. Fluid Mechanics (Pergamon Press, Oxford, 1987) 